\title{
Peer-to-Peer File Sharing in VANETs Using TC-MAC
}

\author{
Mohammad S. Almalag, Stephan Olariu, Michele C. Weigle \\ Department of Computer Science \\ Old Dominion University \\ Norfolk, VA 23529 USA \\ Email: \{malmalag, olariu, mweigle\}@cs.odu.edu
}

\author{
Samy El-Tawab \\ Department of Integrated Science and Technology \\ James Madison University \\ Harrisonburg, VA 22807 USA \\ eltawass@jmu.edu
}

\begin{abstract}
Vehicular Ad Hoc Networks (VANETs) are a form of Mobile Ad Hoc Networks (MANETs) in which vehicles on the road form the nodes of the network. VANETs provide several services to enhance the safety and comfort drivers and passengers. One of the services that VANETs can provide is file sharing between vehicles. Sharing large scale files through the network is a challenging task. Therefore, developing an efficient peer-to-peer (P2P) file sharing scheme is important. This paper presents a P2P file sharing scheme for VANETs using TC-MAC, a MAC algorithm for vehicular ad-hoc networks using Time-Division Multiple Access (TDMA) slot reservation based on clustering of vehicles. Using TDMA will reduce collisions and packet drops in the channel, as well as provide fairness in sharing the wireless medium and minimizing the effect of hidden terminals. Our work aims to develop a P2P file sharing algorithm to improve the file downloading time between neighbouring vehicles.
\end{abstract}

Keywords-Ad-hoc network; Medium Access Control; Vehicular Ad-hoc Network;

\section{INTRODUCTION}

Vehicular Ad Hoc Networks (VANETs) are an important component of Intelligent Transportation Systems (ITS) [1]. VANETs enable the exchange of messages between vehicles and between vehicles and infrastructure. Such communications aim to increase safety on the road, improve transportation efficiency, and provide comfort to drivers and passengers. Figure 1 shows a typical vehicular communications scenario of the future.

In the US, VANETs use $75 \mathrm{MHz}$ of spectrum in the range of 5.850 to $5.925 \mathrm{GHz}$ specially allocated by the U.S. Federal Communications Commission for Vehicle-toVehicle communication (V2V) and Vehicle-to-Infrastructure communication (V2I) using Dedicated Short Range Communication (DSRC) technology [2]. The spectrum band is divided into seven $10 \mathrm{MHz}$ channels (Figure 2). Channel 178 is the control channel $(\mathrm{CCH})$, which is used for beacon messages, event-driven emergency messages, and service advertisements. The other six channels are service channels (SCH) to support non-safety applications.

The IEEE is developing the 1609 family of standards for the Wireless Access in Vehicular Environments (WAVE) [3]. In WAVE, IEEE 1609.4 [4] operates on top of IEEE $802.11 \mathrm{p}$ in the MAC layer. IEEE 1609.4 focuses on the multi-channel operations of a DSRC radio. There is a sync interval (SI) of length $100 \mathrm{msec}$ that consists of a $\mathrm{CCH}$ interval (CCHI) and a SCH interval (SCHI), each separated by a guard interval, as shown in Figure $3^{1}$. During the CCHI, all radios must be tuned to the $\mathrm{CCH}$ to broadcast updates and listen for messages from neighbours and road-side units (RSUs). During the SCHI, vehicles may tune to the SCH of their choice depending on the services offered. The reason for having the length of the SI equal to $100 \mathrm{msec}$ is so that update messages from vehicles are broadcasted at least once every $100 \mathrm{msec}$.

Although the primary purpose of VANETs is to increase safety on the roads by running safety applications, e.g., cooperative collision warning, VANETs can also support several non-safety applications, from notifications of traffic conditions to file sharing. Unfortunately, it has been shown that using DSRC, VANETs cannot support both safety and non-safety applications with high reliability at high traffic densities [5]. Either safety applications or non-safety applications must be compromised.

In this paper, we propose a peer-to-peer (P2P) file sharing scheme for VANETs. Our work will decrease the download time for files between two vehicles, while meeting the requirements for the delivery of safety messages. Our scheme uses TC-MAC as the MAC protocol. TC-MAC uses TDMA [6] to assign a unique time slot to each vehicle.

The rest of the paper is organized as follows. Section 2 gives a background of file sharing techniques in VANETs. Section 3 describes our scheme in detail. Section 4 discusses the evaluation. Finally, Section 5 concludes the paper and presents future work.

\section{RELATED WORK}

P2P systems in VANETs have been a hot topic in recent years. A number of the proposed systems for P2P rely on either on an existing (or imaginary) infrastructure or the cellular system. Abuelela et al. [7] introduced a zeroinfrastructure peer-to-peer system for VANETs (ZIPPER). ZIPPER is designed mainly to support multimedia streaming

\footnotetext{
${ }^{1}$ All radio devices are assumed to be time-synchronized using Global Positioning System (GPS)
} 


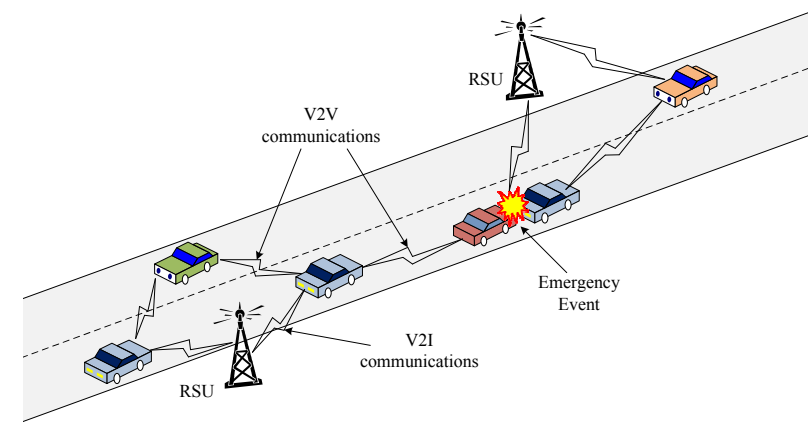

Figure 1. Safety and non-safety communications over DSRC. It shows Vehicle-to-Vehicle communication (V2V) and Vehicle-to-Infrastructure communication (V2I).

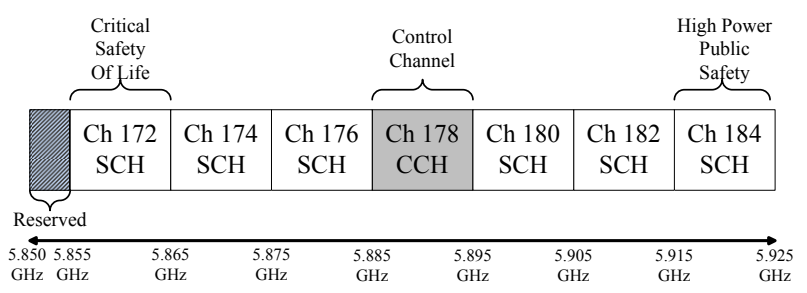

Figure 2. US DSRC spectrum allocation.

such as movies and music, in VANETs. In CarTorrent [8], a work that extends the BitTorrent protocol to vehicular network scenarios, addressing issues such as intelligent peer and piece selection given the intermittent connectivity to preinstalled access point was proposed. Lee et al. [8] have implemented and deployed CarTorrent on a real VANET, which is the first implementation of a content sharing application on a real vehicular ad hoc test-bed. However, given hundreds of highways miles at which there is hardly enough budget to maintain and install lights on the roads, installing gateways every 2-10 miles will be very expensive and not a practical solution. Liu et al. [9] proposed Mobile Chord (MChord), an enhancement of P2P performance over VANETs.

Various types of applications that work on P2P systems can be implemented in VANET since P2P is a powerful platform for a variety of multimedia streaming applications over the Internet, such as video-on-demand, video conferencing and live broadcasting. Hossain et al. [10] performed a case study of a P2P video conferencing system in VANET. Hossain et al. distinguishes between active and passive participants and enhances the video quality of the active participant.

In PAVAN [11], the cellular network is used to broadcast a file description to all vehicles in a certain area. If a vehicle is interested in a file, a route should be discovered and maintained between it and the owner of the file. Scalability is an issue in PAVAN. As the number of vehicles increases, the cellular network cannot handle all the requests and the load of transmission.

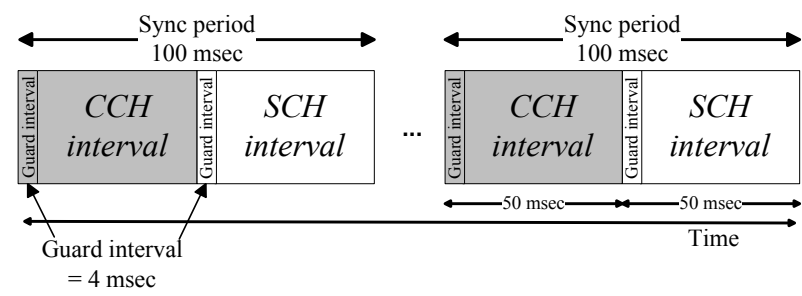

Figure 3. Division of time into $\mathrm{CCH}$ intervals and $\mathrm{SCH}$ intervals, IEEE 1609.4 standard.

\section{OUR SCHEME}

We propose a P2P file sharing scheme for VANETs on top of the TC-MAC protocol [6]. In this section, we will give an explanation of TC-MAC first, and then we will explain our P2P file sharing scheme for VANETs.

\section{A. TC-MAC Protocol}

TC-MAC is a dynamic TDMA slot assignment technique for cluster-based VANETs. TC-MAC, unlike DSRC, allows vehicles to exchange non-safety messages while maintaining a high reliability level for exchanging safety messages. In this technique, the collision-free intra-cluster communications are managed by the Clusterhead $(\mathrm{CH})$ using TDMA.

The TC-MAC protocol assumes an $N$-vehicle cluster. The transmission time is partitioned into consecutive, nonoverlapping logical TDMA frames. It assumes the existence of $k$ slotted SCHs numbered from 0 through $k-1$. In each $\mathrm{SCH}$, the logical TDMA frames are aligned, i.e. begin and end at the same time. Each logical frame contains $\left\lfloor\frac{N}{k}\right\rfloor+1$ slots numbered from 0 through $\left\lfloor\frac{N}{k}\right\rfloor$. All slots are the same size, and the slot size $\tau$ is known to all vehicles in the cluster. For compatibility with DSRC, we assume that $k=6$.

TC-MAC also assumes one $\mathrm{CCH}$, channel $k$, used by the vehicles and the $\mathrm{CH}$ for disseminating status and/or control messages. As with the SCHs, the TDMA frame on channel $k$ is divided into slots of size $\tau$. Each time slot on the $\mathrm{CCH}$ is divided into $k$ mini-slots used to disseminate status information (Figure 4), such as periodic beacon updates used in safety applications.

By virtue of synchronization, the vehicles know the frame and slot boundaries. The number of vehicles $N$ may change dynamically, and the $\mathrm{CH}$ is responsible for updating $N$ and informing all vehicles in the cluster of the new value of $N$.

Each vehicle in the cluster will receive a local ID between 0 to $N$. The $\mathrm{CH}$ will always have ID 1, while ID 0 is reserved for a virtual vehicle. TC-MAC does not expect all $N$ vehicles in the cluster to be communicating, or active, simultaneously. The $\mathrm{CH}$ keeps a list of all the currently-active vehicles and disseminates this list to all the members of the cluster.

In each logical frame, vehicle $j,(0 \leq j \leq N-1)$, owns: 


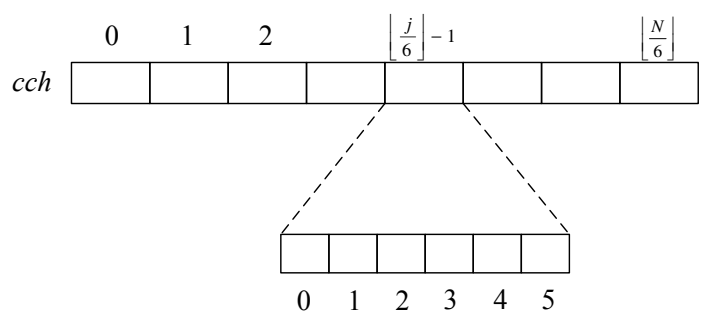

Figure 4. Mini-slots on channel $k(k=6)$; vehicle $j$ owns a mini-slot in the slot preceding its own slot.

- channel $j \bmod k$ during time slot $\left\lfloor\frac{j}{k}\right\rfloor$; we also say that vehicle $j$ owns the ordered pair $\left(j \bmod k,\left\lfloor\frac{j}{k}\right\rfloor\right)$

- the $j$-th mini-slot of slot $\left(\left\lfloor\frac{j}{k}\right\rfloor-1\right) \bmod \left\lfloor\frac{N}{k}\right\rfloor$, on channel $k$, as illustrated in Figure 4; we use the convention that $\left(-1 \bmod \left\lfloor\frac{N}{k}\right\rfloor\right)$ is the $\left\lfloor\frac{N}{k}\right\rfloor$-th slot of the previous logical frame.

The basic idea is that in each logical frame, while idle, vehicle $j$ listens to channel $j \bmod k$ in slot $\left\lfloor\frac{j}{k}\right\rfloor$.

For an illustration of TC-MAC protocol, let $N=61$ and $k=6$. As shown in Figure 5, the vehicle with local ID 39 owns channel $(39 \bmod 6)=3$ during slot $\left\lfloor\frac{39}{6}\right\rfloor=6$, as well as the 4-th mini-slot on the control channel in slot 6-1=5.

For intra-cluster communication in a single-hop cluster, each vehicle uses its own mini-slot to disseminate status information. The first byte of the mini-slot can be used to encode $2^{8}=128$ different situations; a few of them are listed below:

- 0 indicates that the vehicle is not communicating at the moment.

- 1 indicates that the vehicle is involved in communicating with some other vehicle $i$ in the cluster; the binary encoding of the ID of vehicle $i$ follows in the second byte.

- 2 indicates that the vehicle is involved in communicating with a multicast group in the cluster; the binary encodings of the IDs of the members of the multicast group follow in the next 63 bytes.

- $\mathbf{1 2 5}$ is the confirmation of the "handshake".

- 126 indicates that the vehicle will transmit a request during its upcoming slot.

- 127 indicates that the car will use its upcoming slot to transmit.

For point-to-point non-safety communications, setup is done without $\mathrm{CH}$ intervention. Suppose vehicle $i$ wishes to talk with vehicle $j$; setting up a connection between them is done as follows:

- By tuning in to vehicle $j$ 's own mini-slot, vehicle $i$ determines whether or not vehicle $j$ is available.
- If so, vehicle $i$ transmits a handshake packet on channel $j \bmod k$ during time slot $\left\lfloor\frac{j}{k}\right\rfloor$.

Assuming no collision (i.e., some other vehicle may also want to talk to $j$ ), $j$ will pick up the handshake packet and will negotiate with vehicle $i$ the parameters of the data exchange by replying on channel $i \bmod k$ during time slot $\left\lfloor\frac{i}{k}\right\rfloor$; again, assuming no collision, the connection between vehicles $i$ and $j$ has been set up. Now, both vehicles set the first byte of their mini-slots to indicate the status change. Once the connection has been set up, the two vehicles can communicate either during $i$ 's slot, $j$ 's slot, or both.

\section{B. P2P File Sharing Scheme}

The goal of the proposed work is to allow neighbouring vehicles to run non-safety applications such as large-scale P2P file sharing and media streaming services in VANETs. We assume that the vehicle has already found another vehicle that has a file to download. We modified the length of the TDMA frame in TC-MAC to be $100 \mathrm{msec}$. In this case, we guarantee that every vehicle in the cluster sends one update/safety message every $100 \mathrm{msec}$ to meet the safety message requirements.

Suppose vehicle $i$ wishes to share a large file with vehicle $j$; setting up a connection between them is done as follows:

1) By tuning in to vehicle $j$ 's own mini-slot, vehicle $i$ determines whether or not vehicle $j$ is available.

2) If so, vehicle $i$ transmits a handshake packet on channel $j \bmod k$ during time slot $\left\lfloor\frac{j}{k}\right\rfloor$.

3) Since they are sharing a large file, vehicle $i$ will ask a permission from the $\mathrm{CH}$ to use other time slots on the SCHs.

4) $\mathrm{CH}$ will check for unused time slots on the SCHs and grant them to vehicles $i$ and $j$. These granted time slots on the SCHs could be available because no vehicles were assigned to their IDs or because the vehicles assigned to them are inactive.

5) Now, vehicles $i$ and $j$ can start the transmission.

To ensure that vehicles $i$ and $j$ are still receiving update messages from other vehicles nearby during the transmission of the shared file, vehicles $i$ and $j$ will use the granted time slots on the SCHs in the first half of the TDMA frame and then switch to the $\mathrm{CCH}$ during the second half of the TDMA frame. In the following TDMA frame, vehicles $i$ and $j$ will keep listening to the $\mathrm{CCH}$ and then switch to the granted time slots on the SCHs in the second half of the TDMA frame. This process will continue until the file transmission is completed or interrupted by the $\mathrm{CH}$ due to changes in the availability of the unused time slots. An illustration of this is shown in Figure 6.

Because vehicles in the process of transmitting a large file will switch between the two halves of the TDMA frame, they will only hear update/safety messages from other nearby 


\begin{tabular}{|c|c|c|c|c|c|c|c|c|c|c|c|}
\hline & \multicolumn{11}{|c|}{$\leftarrow$ TDMA FRAME (less than or equal to $100 \mathrm{msec}$ ) 1 T } \\
\hline & So & S1 & S2 & S3 & S4 & S5 & S6 & S7 & S8 & S9 & $\mathrm{S} 10$ \\
\hline SCH 5 & 5 & 11 & 17 & 23 & 29 & 35 & 41 & 47 & 53 & 59 & unused \\
\hline SCH 4 & 4 & 10 & 16 & 22 & 28 & 34 & 40 & 46 & 52 & 58 & unused \\
\hline SCH 3 & 3 & 9 & 15 & 21 & 27 & 33 & (39) & 45 & 51 & 57 & unused \\
\hline SCH 2 & 2 & 8 & 14 & 20 & 26 & 32 & 38 & 44 & 50 & 56 & unused \\
\hline SCH 1 & 1 & 7 & 13 & 19 & 25 & 31 & 37 & 43 & 49 & 55 & 61 \\
\hline SCH 0 & 0 & 6 & 12 & 18 & 24 & 30 & 36 & 42 & 48 & 54 & 60 \\
\hline $\mathrm{CCH}$ & $6-11$ & $12-17$ & $18-23$ & $24-29$ & $30-35$ & $36-41$ & $42-47$ & $48-53$ & $54-59$ & $60-65$ & $0-5$ \\
\hline
\end{tabular}

Figure 5. Example for vehicle with ID = 39 in TC-MAC, where N=61 and k=6. The vehicle owns slot 6 on SCH 3 and mini-slot 4 of the slot 5 on the $\mathrm{CCH}$.

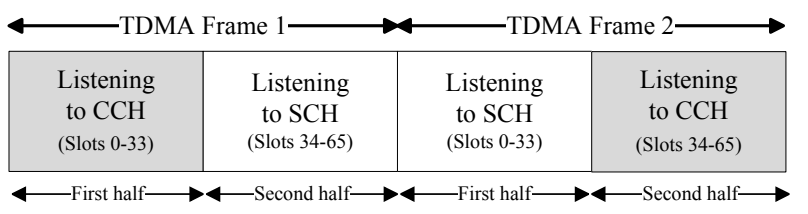

Figure 6. The switching between two halves in one TDMA frame in P2P file sharing. The pair of vehicles that are involved in $\mathrm{P} 2 \mathrm{P}$ file sharing will listen to the $\mathrm{CCH}$ in first half of the TDMA frame 1 and will use the time slots on the SCHs in the second half of the TDMA frame, and vice versa in TDMA frame 2.

vehicles every $200 \mathrm{msec}$. To solve this issue, we need to differentiate between the messages that are missed. If the missed messages are position update messages, the receiver can predict the movement of the sender during this time. On the other hand, if the missed messages are safety messages that are triggered by changes in vehicle behavior, the sender of that message will collect feedback on its recent broadcast message from other vehicles and resend the safety message, if needed. This feedback is done using the Piggybacked Acknowledgement (PACK) protocol [12], which places the following information in each outgoing safety message:

- Sender's position

- The intended range of reception

- A randomly generated message ID

- IDs of most recently received messages (of which this sending node is within their intended ranges)

- The reception time (time earliest) of the earliest message in the acknowledgement list

If vehicle $i$ receives a message $M_{j}$ from vehicle $j, i$ is able to infer feedback on its recently transmitted message $M_{i}$ if and only if two conditions are met: $j$ is within the intended range of $M_{i}$, and the attached time earliest in $M_{j}$ is earlier than the time $M_{i}$ is sent.

For an illustration, assume vehicle $A$ with local $\mathrm{ID}=4$ wants to share a $4 \mathrm{MB}$ MP3 file with vehicle $B$ with local $\mathrm{ID}=15$. Vehicle $A$ will handshake with vehicle $B$ and request time slots on the $\mathrm{SCH}$ from the $\mathrm{CH}$. Assuming that vehicle $A$ and $B$ are granted the requested time slots, the transmission will take place as follows (Figure 7):

- Vehicle $A$ will use (S1, SCH3) to send 1200 bytes to vehicle $B$.

- The $\mathrm{CH}$ will allow vehicles $A$ and $B$ to borrow slots on $\mathrm{SCH} 3$ from other cluster members.

- In order for vehicles $A$ and $B$ to hear the surrounding vehicles, they will use the granted slots from one half of the TDMA frame and alternate with the other half in the following TDMA frame.

- Vehicle $A$ will transfer data to vehicle $B$ using slots from $\mathrm{S} 2$ to $\mathrm{S} 33$ on $\mathrm{SCH} 3$.

- In the following frame, vehicle $A$ and $B$ will use slots from S34 to S64 on $\mathrm{SCH} 3$.

- During slot S65, vehicle $A$ will switch to the $\mathrm{CCH}$ to broadcast update/safety messages to other cluster members.

- During slot S0, vehicle $B$ will switch to the $\mathrm{CCH}$ to broadcast update/safety messages to other cluster members.

With this technique, the total number of slots needed for a file to be transferred from vehicle $A$ to vehicle $B$ is shown in Table I, assuming a slot in SCH can send $1200 \mathrm{~B}$ of data.

\section{EVALUATION}

\section{A. Settings}

The TC-MAC protocol was evaluated through detailed simulation using the ns-3 network simulator [13]. For VANETs, we used modules [14] that added well-known traffic mobility models, the Intelligent Driver Model (IDM) [15] and the MOBIL lane change model [16].

To evaluate our $\mathrm{P} 2 \mathrm{P}$ file sharing scheme, we assume a single-hop cluster, where vehicles can communicate with each other directly. The parameters for the network are listed in Table II. We calculated the download time of a file for one pair of vehicles with different number of slots available for borrowing. The slot availability is based on the number of vehicles in the cluster and their usage of the SCHs. For example, with 32 slots available, we have 195 vehicles $(50 \%$ filled) in the cluster.

\section{B. Results}

TC-MAC performed better than DSRC in delivering safety/update messages. TC-MAC does not experience any 


\begin{tabular}{|c|c|c|c|c|c|c|c|c|c|c|c|c|c|c|c|c|c|c|}
\hline & \multicolumn{18}{|c|}{$\leftarrow$ 个 1 TDMA FRAME $(100 \mathrm{msec})-\rightarrow$} \\
\hline & S0 & S1 & S2 & S3 & $\ldots$ & $\ldots$ & $\ldots$ & S33 & S34 & $\ldots$ & $\ldots$ & $\ldots$ & $\ldots$ & $\ldots$ & $\ldots$ & S63 & S64 & S65 \\
\hline SCH 5 & 5 & 11 & 17 & & & & & & & & & & & & & & & \\
\hline SCH 4 & 4(A) & 10 & 16 & & & & & & & & & & & & & & & \\
\hline SCH 3 & 3 & 9 & $15(\mathrm{~B})$ & BRD & BRD & BRD & BRD & BRD & BRD & BRD & BRD & BRD & BRD & BRD & BRD & BRD & BRD & \\
\hline SCH 2 & 2 & 8 & 14 & & & & & & & & & & & & & & & \\
\hline SCH 1 & 1 & 7 & 13 & & & & & & & & & & & & & & & \\
\hline SCH 0 & 0 & 6 & 12 & & & & & & & & & & & & & & & \\
\hline $\mathrm{CCH}$ & 6-11 & $12-17$ & $\ldots$ & $\cdots$ & $\cdots$ & $\cdots$ & $\cdots$ & $\cdots$ & $\cdots$ & $\cdots$ & $\cdots$ & $\ldots$ & $\cdots$ & $\cdots$ & $\cdots$ & $\cdots$ & $\cdots$ & $0-5$ \\
\hline
\end{tabular}

Figure 7. An example of two vehicles $A(I D=4)$ and $B(I D=15)$ sharing a file under TC-MAC. The first half granted time slots are in green, and the second half are in blue.

Table I

Number of Slots NeEded to Transfer a FILE

\begin{tabular}{|c||c|}
\hline File Size & Number of Slots \\
\hline $4 \mathrm{MB}$ & 3,347 \\
$8 \mathrm{MB}$ & 6,694 \\
$12 \mathrm{MB}$ & 10,041 \\
\hline
\end{tabular}

Table II

TESTING PARAMETERS

\begin{tabular}{|c|c|}
\hline Parameters & Values \\
\hline Cluster Length & $300 \mathrm{~m}$ \\
TX Range & $300 \mathrm{~m}$ \\
Safety Packet Size & $200 \mathrm{bytes}$ \\
Non-Safety Packet Size & $1200 \mathrm{bytes}$ \\
Data Rate & $6 \mathrm{Mbps}$ \\
Mini Slot Size & $0.26 \mathrm{msec}$ \\
SCH Slot Size & $1.52 \mathrm{msec}$ \\
Frame Size & $100 \mathrm{msec}$ \\
Number Slots in the Frame & 65 \\
Shared File Size & $4,8,12 \mathrm{MB}$ \\
Cluster Size & $39,197,316,395 \mathrm{vehicles}$ \\
\hline
\end{tabular}

collisions during the transmission of safety/update messages. For our P2P file sharing scheme, Figure 8 shows that even when all vehicles in the cluster are using their time slots to communicate ( 0 slots borrowed), P2P file sharing still works, but it takes longer time to download the file. This is in contrast to DSRC, which has been shown that it cannot support both safety and non-safety applications with high reliability at high traffic densities. Figure 8 also illustrates how our technique for sharing unused slots improves download performance. Especially for large file sizes, borrowing even just a few slots improves performance dramatically. We note that if the cluster is less than half full, then the second half of the TDMA frame may be used completely for SCH transmission, as no vehicles will be sending on the $\mathrm{CCH}$ at that time.

For an illustration, we will show how we calculated the download time for a file in our P2P file sharing scheme. Assume we have 4 MB MP3 file to be shared between two vehicles in the cluster. Using the network settings in
Table II, the time needed to transfer the file is $5086 \mathrm{msec}$, or $3347 \mathrm{SCH}$ slot times. Based on the size of the cluster, the activity of the cluster members, and the local IDs of the vehicles, the time needed to transfer a file may vary. Assume we have two vehicles, $A$ and $B$, that are on different slot numbers on the $\mathrm{SCH}$ and although the cluster is full, no other vehicles are using their SCH slots. The time to download the $4 \mathrm{MB}$ file is determined by the number of slots needed to transfer the file (3347, as shown in Table I) and the number of usable slots in the frame. There are a total of 66 slots on a SCH, but both vehicles must switch to the $\mathrm{CCH}$ to broadcast during one slot, leaving 64 slots in the TDMA frame for $\mathrm{P} 2 \mathrm{P}$ communication. Due to our technique for listening to the $\mathrm{CCH}$ during half of the TDMA frame (see Figure 6), the number of usable slots is 32. So, with 3347 slots needed to transfer the file and 32 slots available on the SCH each frame, 105 frames are needed to transfer the file. Since each TDMA frame is equal to $100 \mathrm{msec}$, the time needed to download a $4 \mathrm{MB}$ file from vehicle $A$ to vehicle $B$ is $10.5 \mathrm{sec}$.

\section{CONCLusion}

In this paper, we presented a $\mathrm{P} 2 \mathrm{P}$ file sharing protocol using TC-MAC for VANETs. Unlike DSRC, when the number of vehicles that are involved in P2P file sharing is high, vehicles are still able to perform file sharing in each TDMA frame. We also explained the P2P file sharing scheme with examples. The evaluation results show that our scheme is able to share files between vehicles, as well as meet the requirements of safety messages.

In the future, we will further develop our scheme to have P2P file sharing between vehicles in different clusters, with more than one-hop distance. We are also interested in improving upon the Piggyback Acknowledgement protocol to ensure the delivery of safety messages.

\section{REFERENCES}

[1] United States Department of Transportation. Intelligent Transportation Systems. [Online]. Available: www.its.dot.gov/index.htm 

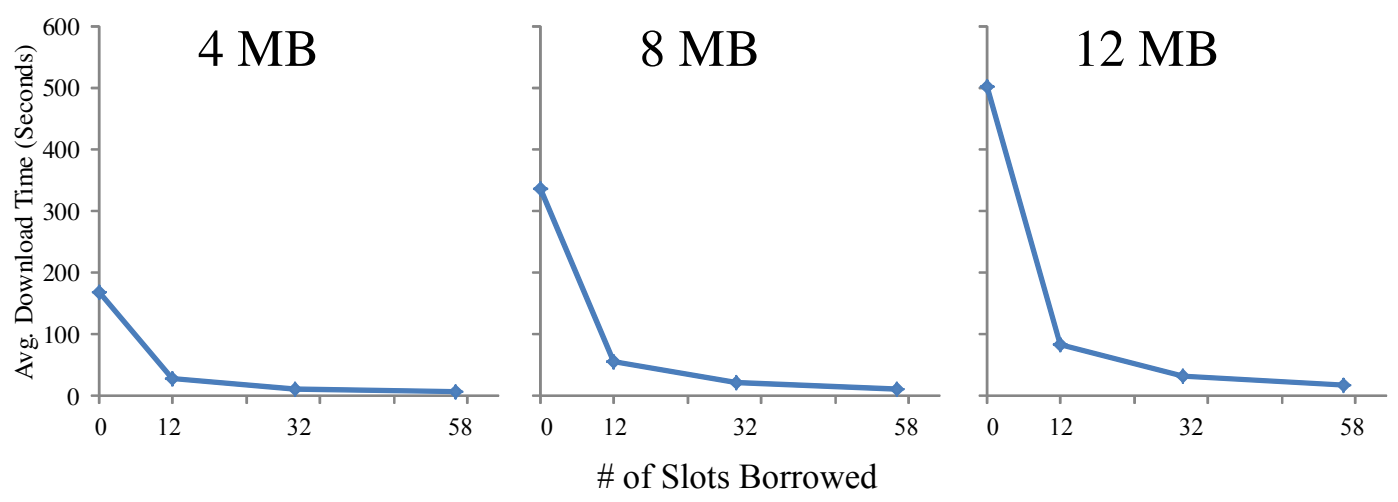

Figure 8. Time to download different file sizes using our P2P file sharing scheme. There are a maximum of 58 slots available for borrowing.

[2] A. E2213-03, "Standard specification for telecommunications and information exchange between roadside and vehicle systems $-5 \mathrm{GHz}$ band dedicated short range communications (DSRC) medium access control (MAC) and physical layer (PHY) specifications," ASTM International, Jul. 2003.

[3] "IEEE Draft Guide for Wireless Access in Vehicular Environments (WAVE) - Architecture," IEEE Std 1609.0/D5, Sep. 2012.

[4] "IEEE Standard for Wireless Access in Vehicular Environments (WAVE) - Multi-Channel Operation," IEEE 1609.42010, Feb. 2011.

[5] Z. Wang and M. Hassan, "How much of DSRC is available for non-safety use?" in Proceedings of the fifth ACM international workshop on VehiculAr Inter-NETworking, ser. (VANET), 2008, pp. 23-29. [Online]. Available: http://doi.acm.org/10.1145/1410043.1410049

[6] M. Almalag and M. C. Weigle, "TDMA Cluster-based MAC for VANETs (TC-MAC)," in Proceedings of the IEEE Workshop on VANETs From Theory to Practice (VTP), San Francisco, CA, June 2012.

[7] M. Abuelela and S. Olariu, "Zipper: A Zero-Infrastructure Peer-To-Peer System For VANETs," in Proceedings of the ACM Workshop on Wireless Multimedia Networking and Performance Modeling (WMuNeP), Chania, Crete Island, Greece, Sep. 2007, pp. 2-8.

[8] K. Lee, S.-H. Lee, R. Cheung, U. Lee, and M. Gerla, "First experience with cartorrent in a real vehicular ad hoc network testbed," in 2007 Mobile Networking for Vehicular Environments, May 2007, pp. 109 -114.

[9] C.-L. Liu, C.-Y. Wang, and H.-Y. Wei, "Cross-layer Mobile Chord P2P protocol design for VANET," IJAHUC, vol. 6, no. 3, pp. 150-163, 2010.

[10] T. Hossain, Y. Cui, and Y. Xue, "Vanets: Case study of a peer-to-peer video conferencing system," in Consumer Communications and Networking Conference, 2009. CCNC 2009. 6th IEEE, Jan. 2009, pp. 1 -2.
[11] S. Ghandeharizade, S. Kapadia, and B. Krishnamachari, "PAVAN: a policy framework for content availabilty in vehicular ad-hoc networks," in Proceedings of the 1st ACM international workshop on Vehicular ad hoc networks, ser. VANET '04. New York, NY, USA: ACM, 2004, pp. 57-65. [Online]. Available: http://doi.acm.org/10.1145/1023875.1023885

[12] D. Jiang, V. Taliwal, A. Meier, W. Holfelder, and R. Herrtwich, "Design of $5.9 \mathrm{GHz}$ DSRC-Based Vehicular Safety Communication," Wireless Communications, IEEE, vol. 13, no. 5 , pp. $36-43$, Oct. 2006.

[13] T. R. Henderson, S. Roy, S. Floyd, and G. F. Riley, "ns-3 project goals," in Proceeding of the workshop on $n s-2$ : the IP network simulator, 2006. [Online]. Available: http://doi.acm.org/10.1145/1190455.1190468

[14] H. Arbabi and M. C. Weigle, "Highway mobility and vehicular ad-hoc networks in ns-3," in Proceedings of the 2010 Winter Simulation Conference (WSC), Dec. 2010, pp. 2991 -3003 .

[15] M. Treiber, A. Hennecke, and D. Helbing, "Congested traffic states in empirical observations and microscopic simulations," Physical Review E, vol. 62, no. 2, pp. 1805-1824, 2000.

[16] A. Kesting, M. Treiber, and D. Helbing, "General LaneChanging Model MOBIL for Car-Following Models," Transportation Research Record: Journal of the Transportation Research Board, vol. 1999, pp. 86-94, Dec. 2007. 